\title{
Unexplained Temporal Effects Recorded by The Torsind at Syzygies
}

\author{
Alexander Pugach \\ Main Astronomical Observatory, 27 Acad. Zabolotnoho St., 03680, Kyiv, Ukraine \\ pugach@mao.kiev.ua, pugach@yandex.ru
}

\begin{abstract}
Observations of the torsion balances' and the torsind's behavior at the moments of the solar and lunar eclipses, the transit of Venus, the occultation of Venus by the Moon were performed. It is shown that in the most cases the devices reaction to these phenomena either outstripped or lagged behind the actually observed phenomenon. The statement is substantiated that the torsind reacts to non-electromagnetic radiation of an unknown nature, arising in the outer space surrounding the Earth.
\end{abstract}

Indexing terms/Keywords: torsion balance, torsind, solar eclipse, moon eclipse, Venus occultation/conjunction/transit, temporal effects.

Subject classification: astrophysics

Type (Method): experimental research article

\section{Introduction}

The influence of astronomical syzygies on the nature of certain physical processes has long been known. In the middle of the last century, the anomalous behavior of the pendulum at the time of the solar eclipse was discovered.

The French Nobel laureate Maurice Allais was the first who revealed that a solar eclipse affects the position of a paraconic torsion pendulum the plane of oscillation [1].

The work of Allais motivated other investigators to perform experiments with torsion pendulums during total solar eclipses. Similar experiments were conducted in different years.

During the solar eclipse of 7 March 1970, E. Saxl and M. Allen found out significant rotation anomalies in massive torsion pendulum. Between the onset of the eclipse and its midpoint there is a steady increase in the observed times required for the torsion pendulum to rotate through a given fixed part of its path, involving both clockwise and counterclockwise motions [2].

Observing the solar eclipse on 11.07.1991 T. Kuusela discovered anomalies in the behavior of a torsion pendulum. As the author writes himself: " two small but distinct shifts were observed in the horizontal position of the pendulum wire which were well correlated with the beginning and the end of the eclipse" [3].

An abnormal reaction of the paraconic pendulum was discovered by L. Savrov during the solar eclipse on 11.07.1991, the full-phase band passig through the territory of Mexico. The device registered a sharp change in azimuth of the pendulum oscillation plane. This jump in the position occurred exactly 2.5 min after the beginning of the eclipse [4].

The energy of solar eclipses affects not only the state of the pendulums. There are several experimental studies, the results of which prove that accurate gravimeters clearly react to the solar eclipse. In particular, there are two works of Chinese researchers who performed gravimetric measurements at the time of a total solar eclipse on March 9, 1997. They found two "gravity anomaly valleys" with a near symmetrical decrease of 
about $6 \sim 7 \mu \mathrm{g}$ at the first contact and the last contact. Thus, the connection between gravitational anomalies and the solar eclipse was established $[5,6$,$] .$

A. Iovane, using 2 tiltmeters as horizontal gravimeters, discovered the appearance of a horizontal tidal force during two solar eclipses: 11.08 .1999 and 31.05.2003 [7].

During the partial solar eclipse on Dec. 24th, 1992, time comparisons between several atomic clocks were made: direct comparisons between different clocks at one and the same station, clock transport comparisons, GPS comparisons and LOC comparisons. And it is concluded from the observations that solar eclipse exerts an influence on the rate of atomic clocks [8].

Subsequently, this conclusion was not confirmed by observations of the solar eclipse in 1999 [9].

However the question remained open, because in these two cases two different eclipses were the object of control. One eclipse does not happen exactly like the other.

We do not aim to prove which of the study groups was right. We want to show that during the solar eclipses, there are other temporal effects that are of interest to researchers. Our research is based on observational material obtained with the help of a new device previously not used in science - the torsind.

What a torsind is? The torsind (TORSion INDicator) is a thread-suspended instrument based on the torsion balance principle. Meanwhile the torsind is a special device where a light disk of non-magnetic metal or paper is used instead of the usual linear beam of the torsion balance. In the torsind a hard suspension (quartz or metal) is replaced by a monofilament silk off the silkworm cocoons [10, 11].

The simplified torsind scheme is shown in Fig1.

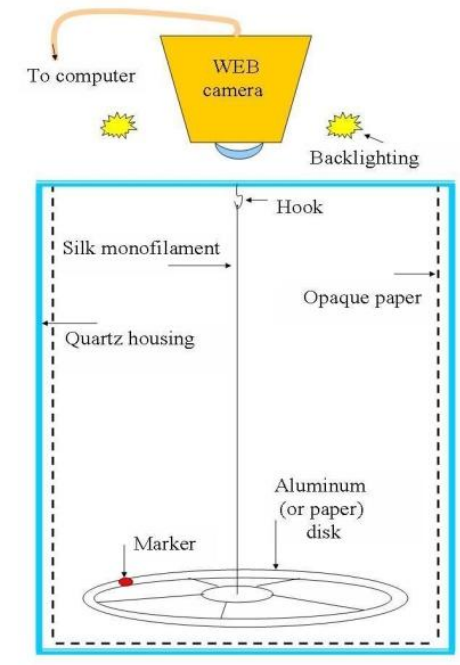

Fig. 1. The torsind has a number of amazing properties that are not inherent to conventional physical devices

The main feature of the device is usage a silk thread because it has no reverse torque when slowly twisted. In many cases, the device's disk rotated by 10, 100, 1000 or more degrees and then remained stationary, not rotating in the opposite direction. Absence of the inverse rotational moment is the specific feature of the silk thread. These experiments were conducted as follows. They were repeated many times and always gave the same result.

The prolonged studies have shown that the following factors cannot be the cause of the significant disc rotation and therefore should be excluded from the further consideration: convective air flow inside the 
torsind housing; changes in outdoor weather parameters (temperature, humidity, pressure); tidal effects of the Moon and the Sun; change in the excitation degree of the ionosphere over the place of observation; Coriolis acceleration; floor vibration; local mobile phone interference. In addition, the torsind is not sensitive to weak and moderate magnetic and electric fields.

These and some other features of the torsind are described in detail in $[10,11]$. All of the above features make the torsind suitable for carrying out non-conventional physical and astronomical phenomena.

The most of the following observations were made mainly in Kiev (Ukraine) at the Main Astronomical Observatory of the National Academy of Sciences. A small part of the observations was made in Romania in the underground salt mine of Cacica. These cases are specified separately. Until 2009 our observations were carried out with the usual asymmetrical torsion balances on the silk filaments which are described in. The systematic torsind observations began in January 2009. Herein we have put together the description of the results obtained with both asymmetrical torsion balances and torsinds as some structural difference of the methods did not lead to significant divergence in the results. The results of these observations are below.

\section{MATERIALS AND METHODS}

\section{Solar eclipses}

1.1. The world's first observation with a group of quasi-identical torsion balances (not torsinds) took place on the night of November 23-24, 2003 during the total solar eclipse, the Totality Path covering the Antarctic coast. Consequently, the eclipse was not visible in Ukraine. Nevertheless, four devices, standing in a room on the 5 th floor side by side, showed the correlated response. The measurement results are presented graphically in Fig. 2. The arrows T1 and T2 indicate start and termination of the eclipse correspondingly. Hereinafter the abscissa is the Universal Time in hours and the vertical axis is the rotation angle of the armpointer (or a disc) with respect to the conditional zero.

Observations began at $2 \mathrm{~h} 15 \mathrm{~m}$ before the actual eclipse starting. Throughout $4 \mathrm{~h} 55 \mathrm{~m}$ all four torsion balances showed the quasimonotonic rotation counter-clockwise. The eclipse developed, climaxed and began to wane and only after that all the instruments began to respond by turning the armpointers clockwise. The beginning of the instruments reaction was belatedly relative to the real signal for more than two hours and 35 minutes.

Solar eclipse on 23-24.11.2003

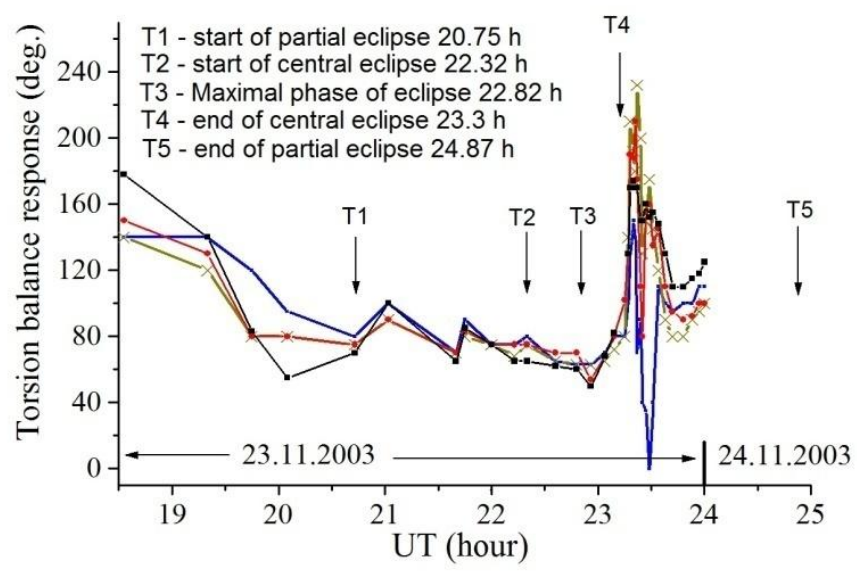

Fig.2 This experiment involving several quasi-identical devices showed that the start of the correlated responses of four instruments to the solar eclipse occurred with the time delay of about 130 minutes.

Observations of the solar eclipses in the following years revealed a number of additional interesting features. 
1.2. When observing the total solar eclipse on March 29, 2006, six neighboring torsion balances showed the clear reaction to this phenomenon, with the maximum response of the instruments being observed at the moments close to the moment of the eclipse maximum phase. Unfortunately, the instruments responses turned out to be less pronounced than in the previous cases. On the broad maxima, it was difficult to determine the exact time of the maximum reaction. If the discrepancy (i.e., delay or anticipation of the reaction) existed, it did not exceed 5-10 minutes and, in addition, different instruments had different discrepancy.

1.3. On September 22, 2006 the annular solar eclipse took place in the Atlantic Ocean and was not visible in Ukraine. Six similar torsion balances for 6 hours before the eclipse were not showing the significant reaction but then almost simultaneously started to react to the eclipse in about 40 minutes after the full phase. Wherein the maximum responses of the devices were delayed an average of 1 hour 6 minutes relatively the maximum phase of the eclipse (see Fig.3). It is worth noting that different instruments had different delay times which varied from 48 to 81 minutes.

Thus, in this case we are also dealing with the explicit delay in the response of the instrument reactions with respect to the moments of the beginning of the eclipse and its maximum phase.

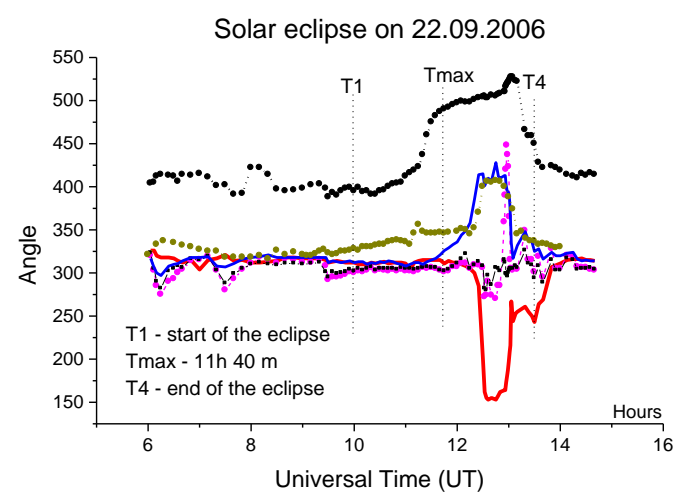

Fig. 3 It is interesting that the maximal instrument responses coincided approximately with the time when the Moon's shadow crossed in Atlantic Ocean the meridian of Kiev.

1.4. On the first of August, 2008 another total solar eclipse was recorded using five torsion balances of the same type. As in the previous case, the behavior of the instruments was relatively calm for a few hours before the eclipse. The devices F11 and F15 did not show any significant reaction throughout the entire solar eclipse. Therefore, we exclude them from consideration and dwell on the analysis of the three remaining instruments' behavior.

The weak response of the instruments began in approximately 30-40 minutes prior to the eclipse start T1. The strong response of the three instruments started near the time of the maximum phase of Tmax (lag of about 63 minutes). And the maximum response of the devices was observed in 33 minutes after the midpoint of the eclipse. Thus, in this case we also encountered the delay response of the device to approximately $33 \mathrm{~m}$ (on average in the group).

Our observations showed that at $(13 \mathrm{~h} 00 \mathrm{~m} \pm 2.5 \mathrm{~m})$ UT i.e. after termination of the eclipse the pointers of all five torsion balances abruptly began to rotate. This is clearly seen in Figure 4. All five instruments, including those two instruments (F11 and F15), which until that time did not show significant deviations, came into motion clock-wise. These movements occurred simultaneously in terms of the temporal resolution of observation. 
This sharp disturbance suggests some abrupt new signal. It does not seem to have been provoked by any local factor including meteorological conditions.

Moreover, the experimental environment remained exactly what it was during the previous 8 hours of observations. It is obvious that this sudden jump was related to the solar eclipse even though it occurred about 4 hours after the first contact. At no other time we ever observed such an abrupt deviation.

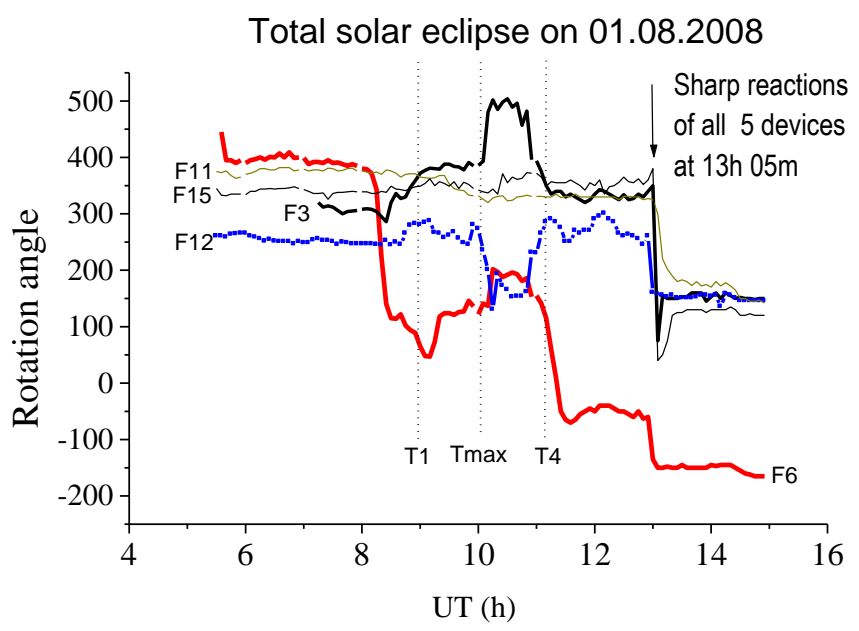

Fig. 4 On this day, there was another important event which description should be discussed in detail.

At the same date a team of researchers conducted coordinated the pendulum experiments in Suceava, northern Romania on the occasion of the August 01, 2008 eclipse.

The Romanian investigators operated two short ball-borne pendulums (one - automatic, other - manual) both of the length about $1 \mathrm{~m}$ and one conventional long the Foucault-type pendulum of the length about $17 \mathrm{~m}$. The "paraconical" pendulum is a solid or physical pendulum suspended upon a small ball which rolls upon a plane and thus has three degrees of freedom: two orthogonal directions of oscillation, and rotation about the vertical axis.

It were the world's first simultaneous observations of the solar eclipse carried out with three different pendulums (in Romania) and five torsion balances (in Ukraine) during which the strange signal was recorded.

When the abrupt sharp signal in Kiev was registered, at the same time both the automatic and manual pendulums in Suceava (Kiev and Suceava are separated by about $440 \mathrm{~km}$ ) changed their oscillation plane. 


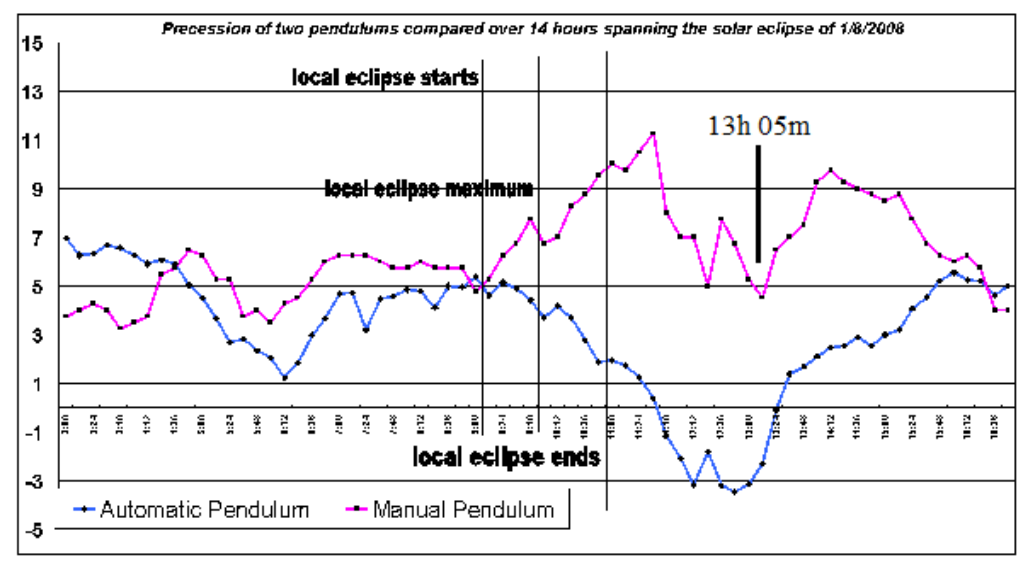

Fig. 5 shows the behavior of both the manual pendulum and the automatic pendulum over the period of 14 hours spanning the eclipse. The abscissa is Universal Time coordinate while the ordinate shows the oscillation planes deviation of the both pendulum relatively any middle position in degrees. One unit on the vertical scale corresponds to $0.64^{\circ}$. The vertical lines in the graphic shows 3 main phases of the eclipse: start, midpoint and end.

The outstanding feature of the results is that, although the types of apparatus used were quite different, in all cases, for each apparatus, the most outstanding peculiar effect was seen after the visible eclipse had ended. Although the nature of the signal described remains the main mystery, the delay in the devices response to the eclipse is still of not less interest. This was not the pattern that might be expected beforehand.

The recorded signal was not of the gravitational nature since the torsion balances are not sensitive to the gravipotential changes. The detailed description of the results is discussed in the separate publication [12]. It marked beginning of the complex observations series with simultaneous use of the pendula and the torsion balances (see below). There is currently no adequate explanation for this strange signal.

1.5. The first observations of the partial solar eclipse with a primitive automatic torsion balance (quasi-torsind) took place on 11.09.2007.

The automatic balance based on the principle of shaded LEDs gave the low counts accuracy of about $5.6^{\circ}$. However, it possessed the advantage that it relieved the operator of the tedious procedure of visual counts reading. This first specimen of the automatic quasi-torsind was operated continuously for more than 66 hours from 10.09.07 up to 13.09.07.

The result of these continuous observations is shown in Fig. 6 . The vertical arrows, as usual, show the moments of the three main phases of the eclipse. The result obtained is of interest from the point of view of the anomalous temporal effects study. The first 17 hours of measurements showed no significant deviations with the exception of the small fluctuations within $\pm 6^{\circ}$. But suddenly at 13 o'clock KT, i.e. 25 minutes before the beginning of the eclipse, the instrument armpointer made a 30-degree excursion counterclockwise and then returned to its original state. 


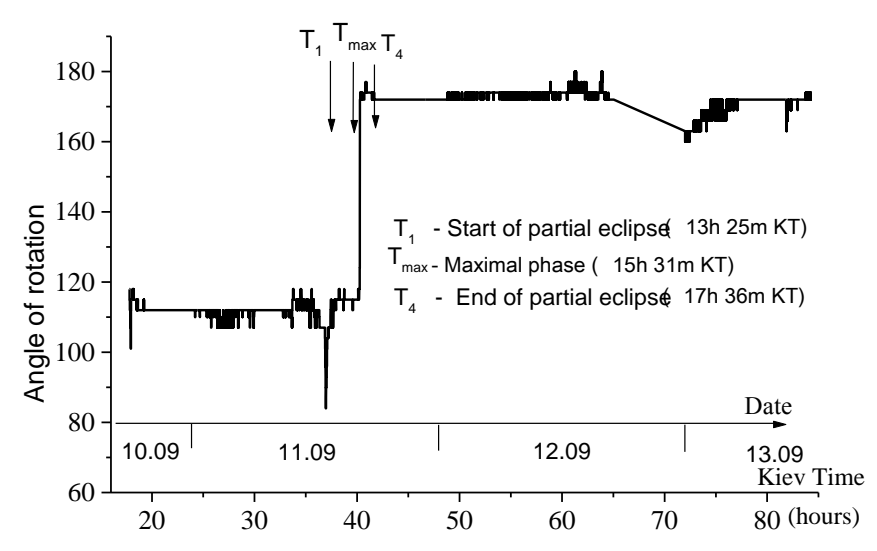

Fig. 6 The next jump of the armpointer occurred 2 hours 52 minutes after the eclipse began. This time the pointer turned 55 degrees clockwise and then remained practically motionless for the next 24 hours. Thus, in this case we also encountered an example of the fact that during the solar eclipses the torsion balance displays activity at times that do not coincide with the moments of the beginning, the end, and the maximum phase of the eclipse.

1.6. On January 26, 2009 the simultaneous observations of the two torsinds' (Kiev, Ukraine) and the two paraconical pendulums' (Suceava, Romania, $440 \mathrm{~km}$ away) reactions were performed during the solar eclipse that was not visible at those locations but in the Indian Ocean only.

Five days before the eclipse, two nearly identical torsinds WEB-1 and WEB-2 were installed at Main Astronomical Observatory NASU in an insulated, heated, and dry room with tightly closed windows and doors. The measurements began on January, 23 at $17 \mathrm{~h}$ UT and lasted about 6 days without interruption, up to $23 \mathrm{~h}$ $59 \mathrm{~m}$ UT on January 28 . Thus, the reaction of the torsinds to the surrounding circumstances was tracked for about 3 days before the eclipse and 2.5 days after. The eclipse on Earth began on January, 26 at 4h 56m UT and ended at $11 \mathrm{~h} 00 \mathrm{~m}$ UT. The geocentric conjunction of the Sun and the Moon in right ascension took place at $8 \mathrm{~h} 06 \mathrm{~m}$ UT. The results of the torsind observation are partially presented in fig. 7 in blue (WEB-1) and purple (WEB-2).

Thoughtful analysis shows that these readings of WEB-1 and WEB-2 tightly correlate. Calculations show that, in this time interval, the correlation coefficient $C_{(\text {WEB-1/WEB-2) }}=0.892$. Such the high value of $C C$ indicates that the reactions of the WEB-1 and WEB-2 were not random and there is some common cause that affects the torsinds. This conclusion is confirmed by the fact that such correlation is absent outside of the eclipse time interval T1-T6.

Two paraconic pendulums (PP) used in the observations were installed in the basement of the planetarium of Stefan cel Mare University, Suceava, Romania. The measurements with PP began almost 7 hours before the solar eclipse. The results obtained with these two pendulums, one was monitored manually and the other automatically, were identical. They are presented in Fig. 7 in red.

Comparison of the PP measurements data with the results of the torsind revealed the amazing and important coincidence. From the preliminary visual comparison of the results, it appeared that the torsind graph in the interval T1-T6 almost exactly repeated the pendulums graph being shifted along a time coordinate. In other words, the event in Suceava anticipated the event in Kiev. 
In order to clarify the value of this temporal advance, the crosscorrelation analysis of the two types of measurements (torsind and PP) was carried out. The results of the calculations are shown in Figure 8. The crosscorrelation function reaches the maximum at the correlation radius of $1.59 \mathrm{~h}=95.4$ minute.

That is, the best match between the graphs of the PP and the torsind will be if one curve is shifted in time relative to the other by 95.4 minutes. After such treatment the correlation coefficient for the PP graph and the WEB-1 graph is equal 0.921 .

In other words, cross-correlation analysis showed that some event that registered the RR occurred in Suceava 1.5 hours earlier than in Kiev. This event was equally reflected by the devices as evidenced by the graphs in Fig. 7 , showed the amazing good coincidence.

Simultaneous torsind \& paraconical pendulum observations

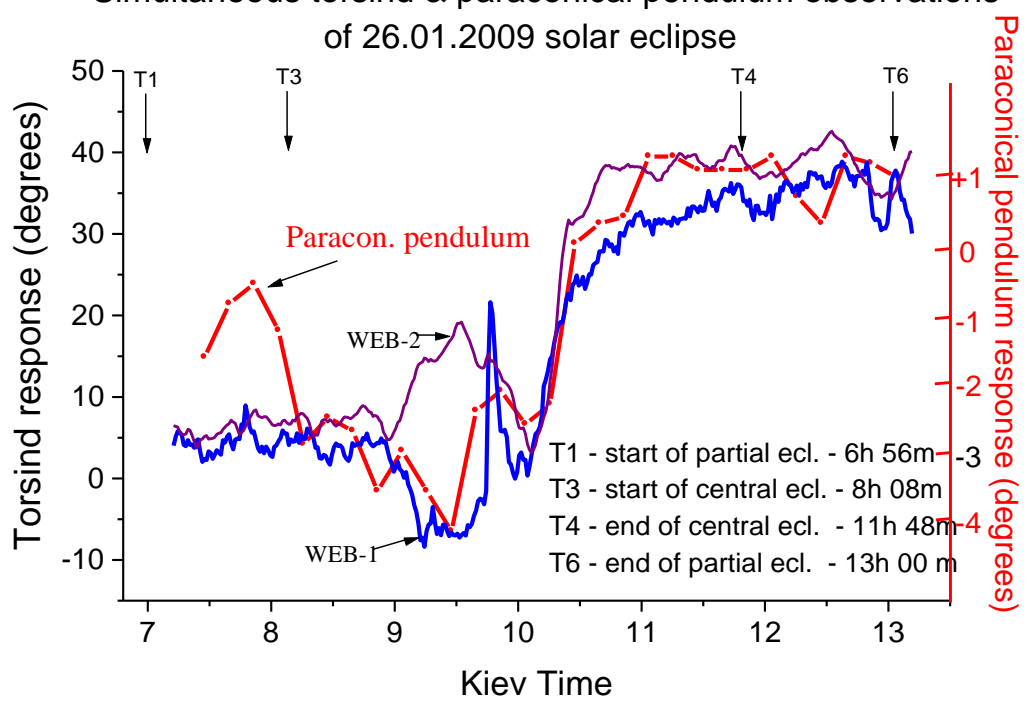

Fig. 7

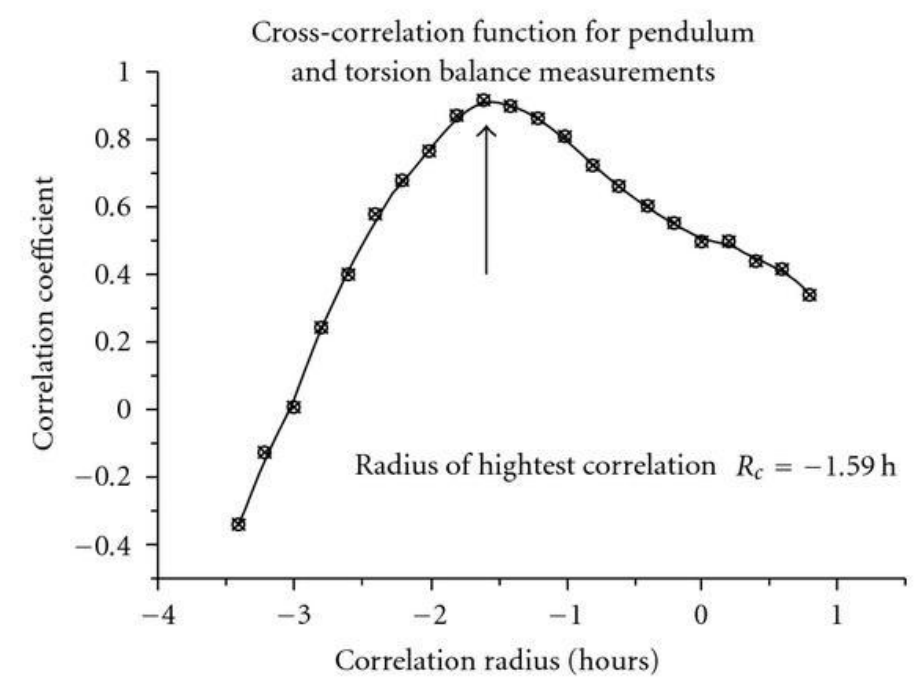

Fig. 8 The above data give grounds to assert that the changes in the azimuth of the paraconical pendulum oscillation plane and the torsinds actions on January, 26, 2009 were not accidental. Such coincidence of the 
different instruments results clearly indicates the presence of some dominating signal and excludes any assumption of accident.

In detail, the results of this study are described in [13].

1.7. The very interesting results were obtained in the complex observations of the solar eclipse on January 15 , 2010 invisible in the observation point.

The observations of this eclipse were carried out in Kiev with the torsion balances whose behavior was monitored by a television camera. The pictures were taken every minute and the images were recorded in the computer memory.

Measurements began on January 14, at 17 hours before the eclipse onset. Until the first contact the device did not register any significant variations. But $1 \mathrm{~h} 19 \mathrm{~m}$ after the first contact there was the armpointer sharp rotation. Within 5 minutes, the armpointer of the device turned at almost 45 degrees CCW and then quickly returned to its original position. There were almost two hours before the maximum phase of the eclipse should come. The peculiarity of this astronomical phenomenon was that the maximum device readouts almost coincided in time with the maximum phase of the phenomenon. The device response lagged behind the maximum moment by only 10-15 minutes. In other cases, as can be seen from the previous description, the instrument readings were quite a bit late in relation to the real phenomenon.

Solar Eclipse on 15.01.2010

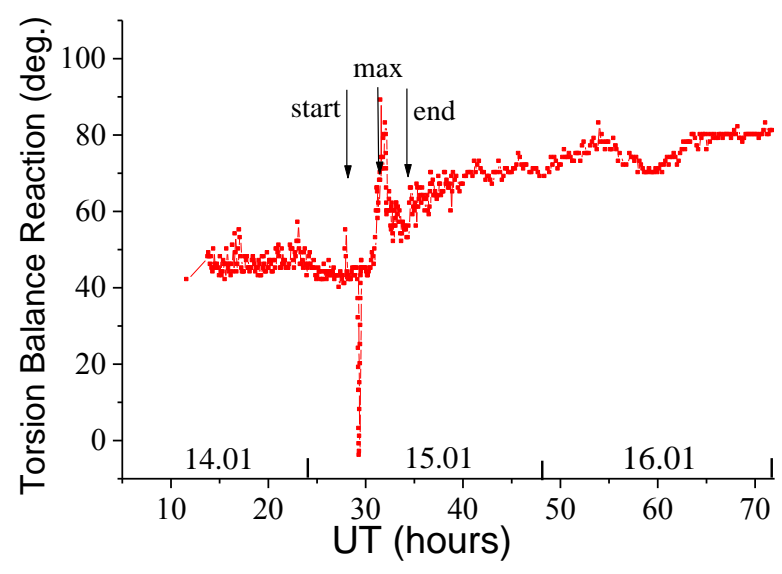

Fig. 9 But in this case, there is still the significant temporary anomaly associated with the negative peak appearance after $1 \mathrm{~h} 19 \mathrm{~m}$ after the eclipse began. Due to this feature, we have included this case in our review. The result of the instrumental observations at this date is shown in Fig. 9. Abscissa is the Universal Time with zero at 00h 00m UT on January 14.

In addition, those who wish to view the television recording of the device's arrow rotation may go to the site [14]. The more detailed description of the observations results can be found in the further section which describes the frequency changing of the rubidium standard associated with the eclipse.

1.8. The interesting results were obtained in the course of the November 13/14, 2012 solar eclipse complex observation. Four instruments were involved. The two torsinds WEB_1 and WEB_2 were operating in Kiev, Ukraine.

The Kiev measurements began 6.5 hours before the maximum phase of the eclipse and lasted continuously for 15 hours. One measurement was taken each minute. The results of the Kiev torsind measurements are shown 
in Fig. 10 in red and blue lines. The dashed vertical lines indicate the beginning and the end of the eclipse on the Earth. This eclipse was not visible in Romania or Ukraine.

The Kiev observations showed good convergence of the results. This is confirmed by the high cross-correlation coefficient which in this case was equal to 0.975 .

Two approximately identical Foucault pendulums operated in Romania. The first Foucault pendulum of $6.43 \mathrm{~m}$ length was installed in the Cacica salt mine, Suceava country, at $75 \mathrm{~m}$ depth. The second one of $6.15 \mathrm{~m}$ length was mounted in a special room in Horodnic $23 \mathrm{~km}$ away from Cacica.

It is shown that during the eclipse both the torsinds and the pendulum exhibited specific reactions: the torsind's disk rotated whereas the direction of the swing plane, the period, the eccentricity, and the chirality of the ellipse of the Foucault pendulum oscillation were all altered.

All these results are represented in one graph as shown in Fig. 10. The dashed vertical lines indicate the beginning and the end of the eclipse on the Earth.

Solar Eclipse, 13-14 of November, 2012

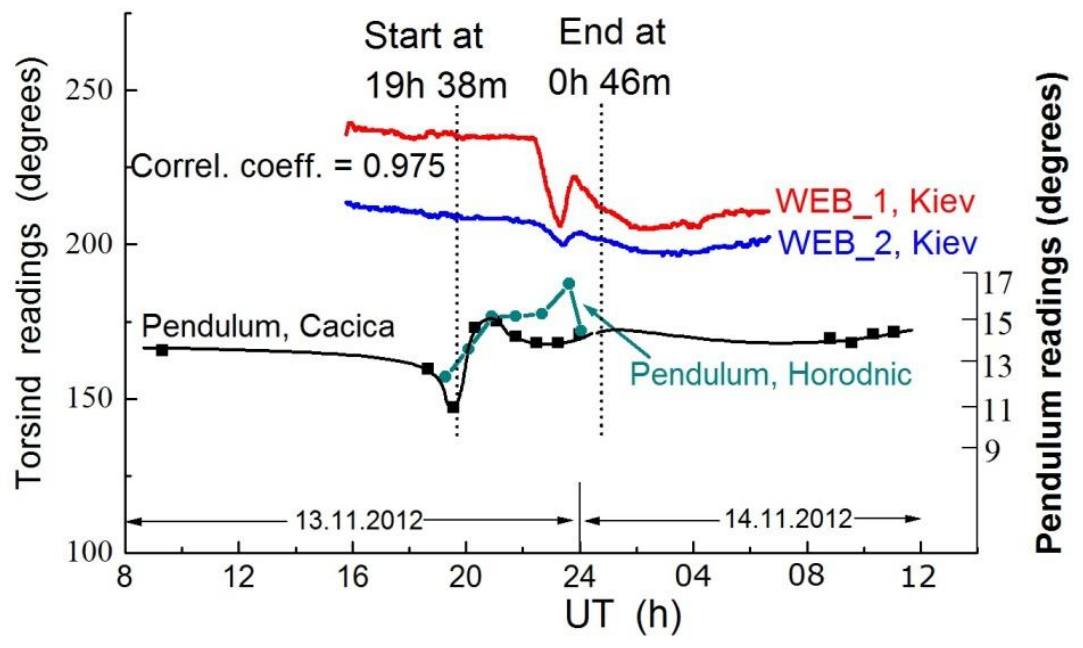

Fig.10 The Cacica pendulum manifested the single great deviation in the counter-clockwise direction at the moment near the eclipse start. This is the interesting event since in some other cases the pre-maximal decrease in counts occurred after the eclipse began. But the maximum deviation of the oscillation plane of the Cacica pendulum occurred only after 86 minutes.

The Horodnic pendulum showed the significant deviation at the moment after the eclipse midpoint. At that time two Kiev torsinds displayed the synchronous diminutions. Obviously, these events are causally related and the solar eclipse is the main reason. The detailed description of these results may be found in [15].

1.9. In 2013, the complex observations were made on May 10 during the annular solar eclipse using two pendulums and three torsinds.

This time the torsind W4 was set in the salt mine of Cacica at the depth of about 40 meters, the torsind W5 in Comanesti village, and the torsind WEB_1 was in Kiev. Measurements with the pendulums were performed in the same format as in 2012. 


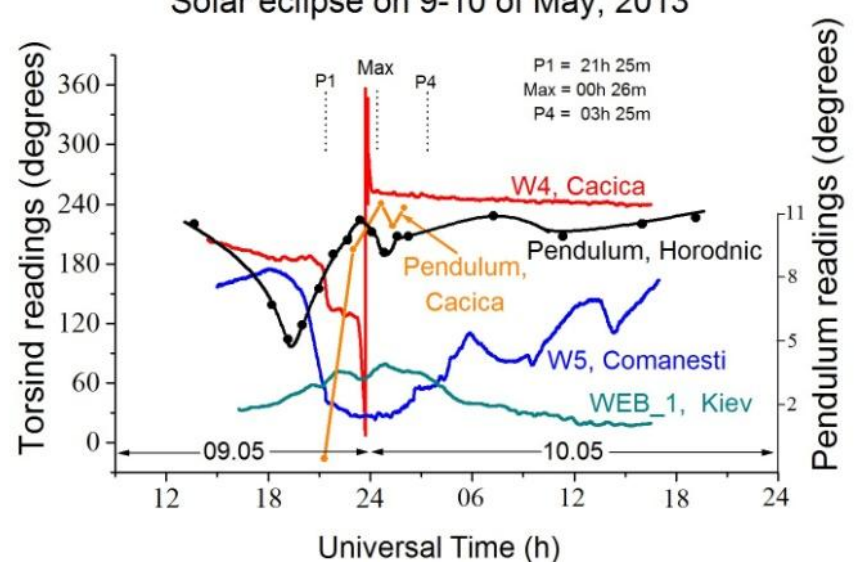

Fig.11 All the instruments showed the significant response with the maximum counts observed in the interval between the beginning and termination of the eclipse (See. Fig. 11). It is of a particular note that the maximal Foucault pendulum reaction was a few hours ahead of the torsind reaction as it was in the previous year. The description of these experiments detailing the pendulum measurements is presented in [15].

The Final results of the Section 2 can be formulated as follows.

- The torsind "feels" solar and lunar eclipses even when the astronomical event occurs on the reverse side of the globe and is not visible at the place of observation. The torsind reacts to a signal which seems passing through the Earth. In this case, it can be argued that the torsind reactions have nothing to do with electromagnetism.

- The maximum response of the pendulums does not coincide in time with the maximum of the eclipse phase. Such mismatch indicates that gravity can't be the cause of the pendulum and the torsind reactions.

- It is possible that the investigated time shifts between the cause and the result are possible namely because in these phenomena we are encountering a new kind of radiation.

\section{MOON ECLIPSES}

Three lunar eclipses were subjected to observations for all the work time with the torsion balances and the torsinds. Two of them - on October 17, 2005 and March 14/15, 2006 showed the similar reaction. As in the solar eclipses case, the maximal instrument response in these cases did not coincide in time with the maximum of the eclipse phase. Here we do not dwell on their detailed descriptions

2.1. It was the special interest for the third of the observed lunar eclipses which took place on February 9 , 2009. Two days before it, i.e. on February 7 and 8, and within three days after the end of the eclipse (February 10,11 and 12) the baseline monitoring was conducted. It made possible to find out what was the nature of the torsion balances reaction in the days outside the eclipse and to compare this reaction on the eclipse day. In Figure 12 the background records are shown in black. The record of the lunar eclipse is represented in blue. 


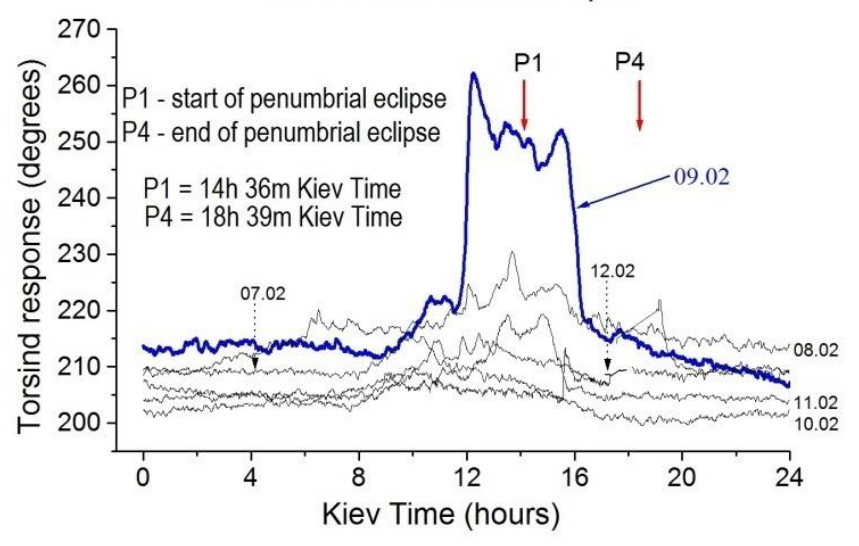

Fig. 12

The clear torsind response on the day of the eclipse is visible well. The amplitude of the peak on the date 09.02 was approximately 6 times more the average amplitude on the days outside the eclipse. The torsind

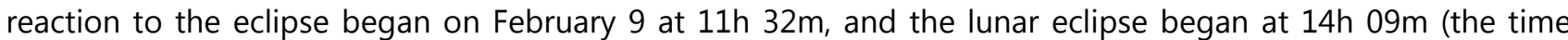
shift $=2 \mathrm{~h} 37 \mathrm{~m}$ ). The beginning of the reduction of the torsind countdowns took place at $15 \mathrm{~h} 30 \mathrm{~m}$, and the end of the eclipse was at $18 \mathrm{~h} 25 \mathrm{~m}$ (the shift $=2 \mathrm{~h} 55 \mathrm{~m}$ ). So, we can say that the width of the reaction and the width of the eclipse itself were approximately the same. And on the average the reaction of the torsind preceded the astronomical event by approximately $2 \mathrm{~h} 45 \mathrm{~m}$

Thus, the results of the lunar eclipses observations raise the questions of these temporal shifts nature. However, the answers to these questions are not known with certainty.

\section{VenUs occultation/Conjunction/Transit}

3.1. The Venus occultation by the Moon was on June 18, 2007. In Kiev the five torsion balances were used simultaneously standing nearby. This phenomenon took place during daylight and the good weather conditions allowed to observe visually the phenomenon in the sky. The binoculars gave an opportunity to clearly see as the Venus and the Moon's discs were approaching to each other. At the moment when the Venus was not yet near the lunar disk, however, all the five devices already responded by turning their armpointers.

The visual measurement results are shown in Fig. 13. It can be seen that a few hours before the eclipse all the five devices were relatively quiet. The significant reaction of all the devices appeared in about 1.5 hour before the first contact T1. The return of the four instruments (F4, F8, F7, F5) to their original states occurred at about 1.5 hours before the phenomenon end. It is obvious that the torsion balances reactions outstripped the natural course of events just as they did it in the case of the Moon eclipse (paragraph 3.1). The width of the reaction band was approximately equal in time to the difference of the moments (T4-T1). 


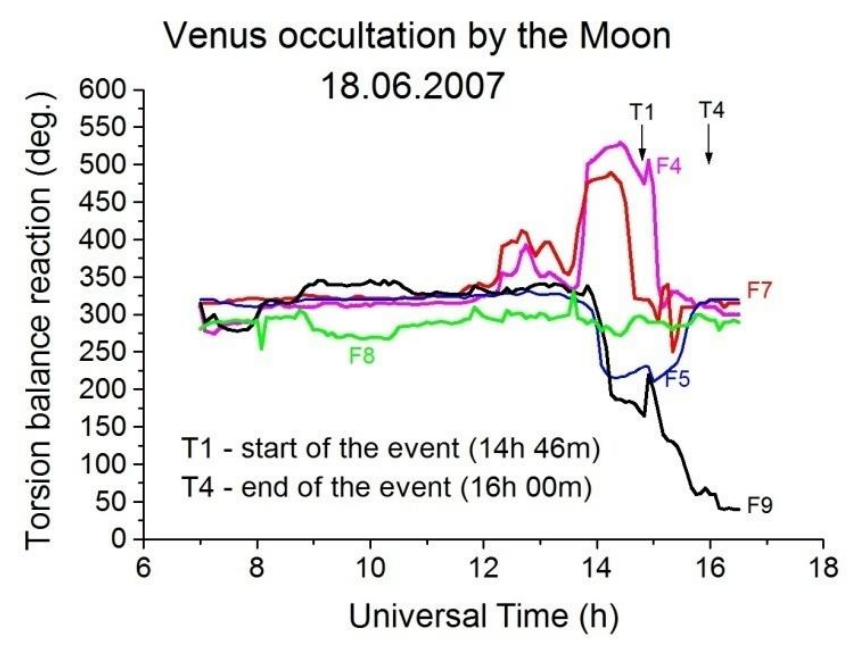

Fig. 13

The average outrunning of the 4 devices was approximately 70 minutes. What a reason may be behind the result? This time-shift can`t hardly be explained from the position of gravity theory.

\subsection{The Venus - Moon conjunction on 16.05.2010.}

The observations were made during three days $(15,16$, and 17 of May). The three devices were involved in the observation process: two torsinds (WEB_1 and WEB_2) and an asymmetric beam torsion balance with a continuous results recording through a TV-camera (KB-TV).

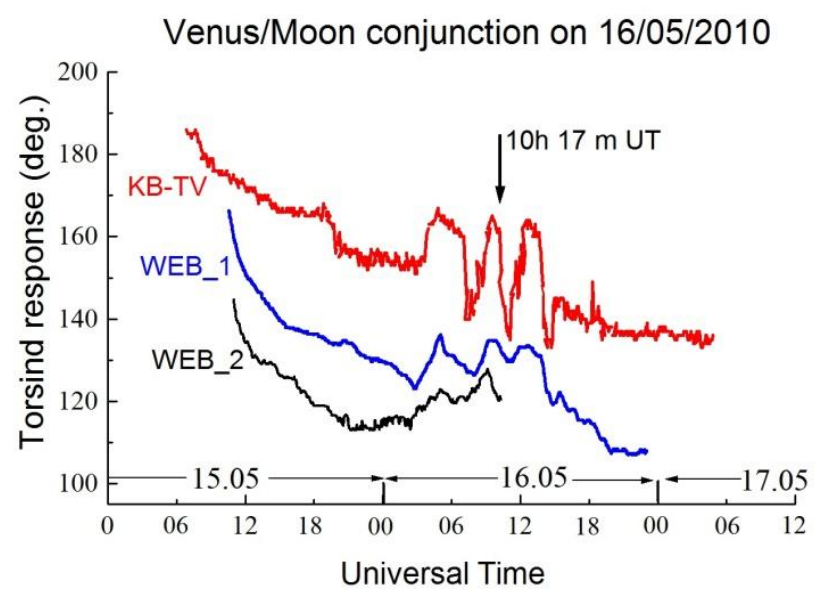

Fig. 14.

The last unit was located in the neighboring building at the distance of about $150 \mathrm{~m}$ from the torsinds. Nobody came into the room, where there were the instruments throughout the measurement period. The result of the observations is shown in Fig. 14. The three waves are imposed upon the descending trend with the middle wave maximum coinciding with the calculated conjunction moment. The vertical arrow indicates the moment of the Venus - Moon conjunction in right ascension. All three devices showed the similar picture. The instruments significant response started at $7 \mathrm{~h} 32 \mathrm{~m}$ before the conjunction and ended $4 \mathrm{~h} 56 \mathrm{~m}$ after it.

It is noteworthy that the correlation coefficient between the curves WEB_1 and KB-TV was quite high and amounted to $\mathbf{0 . 8 7 2}$. This suggests the unquestionable reliability of the obtained result. The similar reaction of the devices installed in different locations indicates the space factor rather than the earthly nature of the recorded signals. 
3.3. The Venus transit. The last transit of the Venus occurred on June 5-6, 2012. We were observing this phenomenon during the 5, 6, and 7 of June using the two identical by construction torsinds (WEB_1 and WEB_2). They were installed in an isolated tower of the idle telescope of the Main Astronomical Observatory in Kiev. Figure 15 shows the results of the observations which lasted during 5, 6, and 7 of June. The first contact Venus-Sun occured at $22 \mathrm{~h} 09 \mathrm{~m}$ UT and the last one - at $04 \mathrm{~h} 49 \mathrm{~m}$ UT on the next day.

Despite the fact that the devices' reactions were somewhat different, both units showed the similar reaction to this phenomenon. As the most important result remains the fact that both torsinds "caught sight" of the phenomenon only a few hours after its starting. It happened only at $5 \mathrm{~h} 48 \mathrm{~m}$ after the first contact when both devices began to increase their readings at the same time [16]

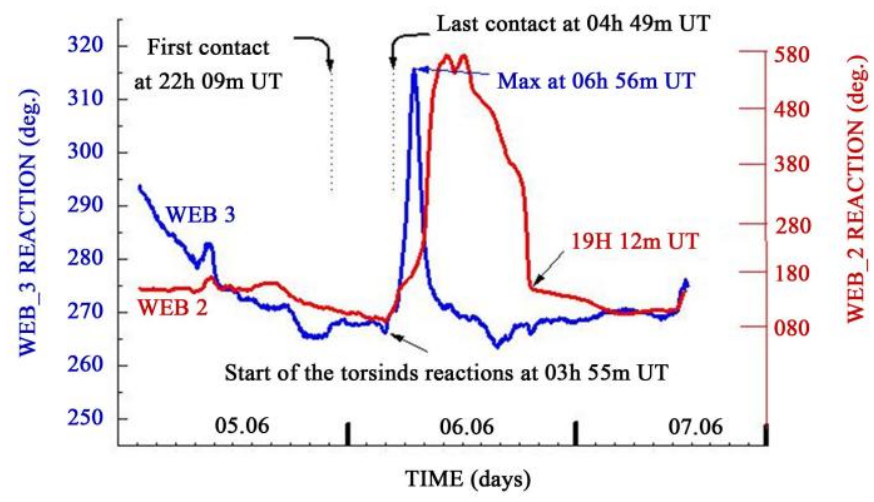

Fig. 15

The delayed reaction of both devices gives grounds to assume that the Venus transit caused some changes in the intensity of the solar radiation component that carries the torque effect. In theory, the existence of this component, called Spiral Vortex Radiation of the Sun (SVRS), was predicted by professor G.A. Nikolsky from St. Petersburg [17]. The Venus transit observations made it possible to determine accurately the speed of SVRS equal to $1990 \mathrm{~km} / \mathrm{s}$. These results are presented in details in [16].

Thus, the torsind observations of the astronomical phenomena associated with the planet Venus allowed to discover a number of new facts that represent a certain difficulty for the current physical theory. In particular, there are the outrunning torsind responses during the Venus occultation by the Moon (Fig. 13) and the conjunction of these celestial objects (Fig. 14) as well as detecting a hypothetical solar radiation that transfers the torque at the speed of about $2000 \mathrm{~km} / \mathrm{s}$.

\section{Rb-STANDARD FREQUENCY SHIFT}

4.1. In 1992, Chinese researchers reported on instrumental tracking of the frequency shift of atomic clocks during the total solar eclipse [5]. They found that at the time of the total phase the atomic clock changed its course. This example inspired us and we decided to do something like this.

During the 15.01.2010 solar eclipse the difference between the local rubidium frequency standard (RbSt) at the observatory and the standard GPS signals were under registration. This RbSt standard is commonly used in Main Astronomical Observatory in the program of satellites laser observations.

In order to tie the chronometric measurements to the eclipse phases, the observations of the torsind behavior began at 17 hours before the astronomical phenomenon onset . 
The result of the eclipse torsind observations is presented in Figures 9 and 16 with red dots. The abscissa is Universal Time with zero at 00h 00m UT on January 14. The eclipse's start and the end are indicated by the arrows $\mathbf{s}$ and $\mathbf{e}$. Within $1 \mathrm{~h} 29 \mathrm{~m}$ after the eclipse start the torsion balance armpointer turned sharply to $46^{\circ}$ counterclockwise and then quickly returned to the original position. The video of this episode is available in [14]. After $2 \mathrm{~h} 5 \mathrm{~m}$ there was digression into the opposite direction as can be seen in the graph. This last excursion ended when the Moon's shadow was still falling onto the Earth's surface.

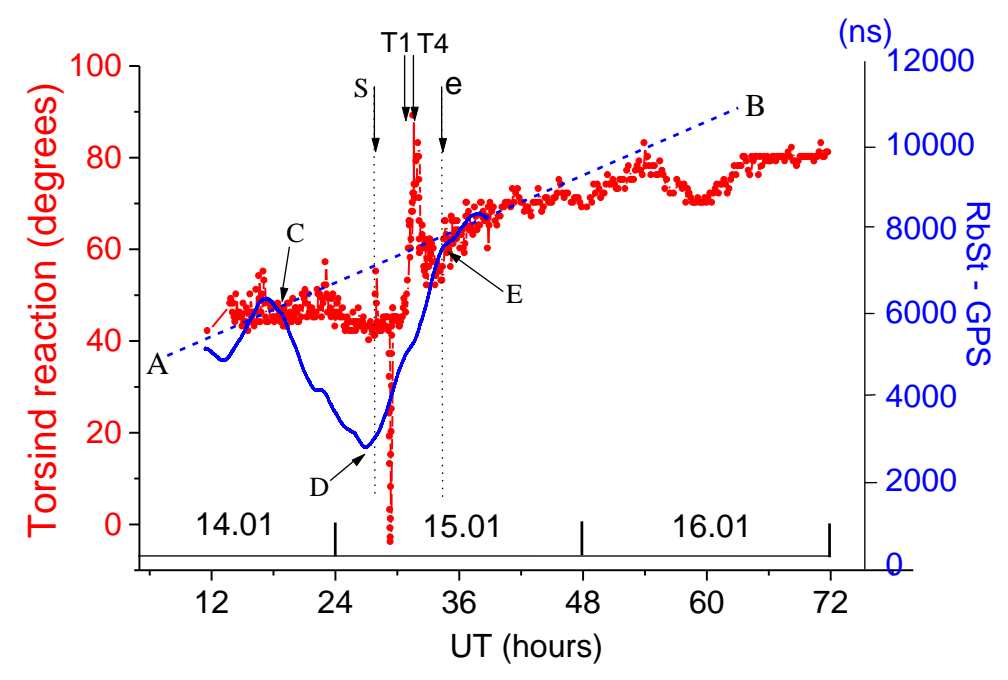

Fig. 16.

The shown curve can be roughly represented as a sequence of the five phenomenon phases:

1- the general trend, i.e. the systematic increase in the counts from the start of the

measurements to the end.

2 - the wide depression in the interval from 23 hours UT to 36 hours UT.

3 - the sharp symmetrical negative peak at about $29.4 \mathrm{~h}$ UT.

4 - the relatively wide asymmetric positive peak in the interval of $32 \mathrm{~h}-34 \mathrm{~h}$ UT.

5 - the second wide depression in the interval $54-63 \mathrm{~h}$ UT.

A very significant result is the detection of the sharp negative jump at the time around 29.4h UT when the armpointer began to turn and within 22 minutes made an excursus against the clockwise and then backwards with the amplitude of about 49 degrees. After 22 minutes, the armpointer came to the same position from which it started. However, the most important thing is that the minimum response of the device occurred 88 minutes after the eclipse had begun and the maximum response occurred 55 minutes after midpoint of the eclipse!

In parallel with these observations, the chronometric measurements under the leadership of Dr. Medvedsky M.M. were made. Their goal was to compare the phases of the high-precision rubidium frequency standard (RbSt) located on the observatory territory with the readouts of the external module of high stable frequency generated by the GPS system. The local RbSt standard was providing the laser station with the high-precision $U$ Time scale. Its long-term accuracy is on the order of $10^{-12}$ seconds. 
As a second source of frequency, the specialized TRIMBLE Thunderbolt E receiver was used, which long-term stability is characterized by the similar accuracy of $10^{-12} \mathrm{~s}$. The phase difference was measured using the time meter SR-620 which has the resolution better than 100 picoseconds. The phase difference was measured at the beginning of every second. The measurements were carried out autonomously under the control of a PC. The measurement result is shown in Fig. 16 by the blue line (for this case the nanosecond scale $10^{-9} \mathrm{~s}$ is built on the right side of the graph).

From the preliminary multi-day observations, it was established that the daily rates of the RbSt standard and the GPS standard did not coincide. Their divergency averages $+141 \mathrm{~ns} / \mathrm{h}$. This value is the slope of the straight line $A B$ shown by the blue dashed line. If the daily rate of the RbSt standard was constant, then the difference (RbSt-FMS) would lie along the line AB. However, this was not the case. The difference " RbSt -GPS" has the systemic deviations from this line.

As early as January 14, almost 10 hours before the start of the eclipse the difference (RbSt - GPS) began to change (see the blue thick line in Fig. 16).

For the ten-odd hours before the eclipse start, the average daily rate changed its sign and on the segment CD (losing segment) it was equal to $366 \mathrm{~ns} / \mathrm{h}$. One hour before the beginning of the eclipse (point $\mathrm{D}$ ), the daily rate again changed its sign (the gaining mode) and on the segment $D E$ it was equal to $+521 \mathrm{~ns} / \mathrm{h}$.

Thus, on the day of the eclipse:

- there was the very strong deviation of the RbSt rate from the GPS one;

- the maximal deviation of the rate was registered 1 hour before the eclipse start and 4.8 hours before the eclipse maximal phase.

Observations of the atomic clocks frequency variation during eclipses was observed by other researchers before us [8]. But the anticipating effect of the anomalous behavior of the frequency rubidium standard is found by us for the first time.

In addition to $\mathrm{MAO}$, chronometric measurements were also carried out at the State Institute of Metrology "Ukrmetrteststandard" (the executor is Dr. Golovnya M.V.), located at $3 \mathrm{~km}$ southwest of the MAO. They lasted a little less than 7.5 hours but they captured almost the entire phase of the eclipse. The phase differences of the quartz frequency standard (Quartz) were compared with the GPS source of the standard signals. The rootmean-square relative variation in the output signal frequency of the Quartz was $2 * 10^{-11}$. These measurements showed qualitatively the same deviation of the difference (Rb-standard minus GPS), as well as the MAO measurements. The time scale is the same for the both measurements. 


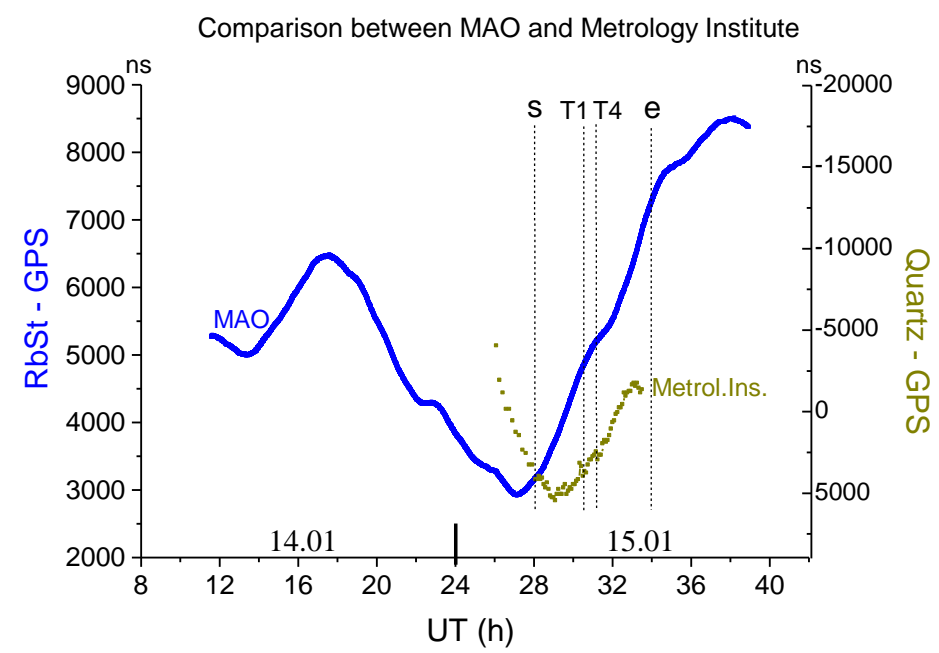

Fig. 17

The comparison is shown in Figure 17. Obviously, the curve "Quartz - GPS" almost identically repeats the curve (RbSt - GPS), but with the delay of 2.5 hours.

This is another temporal effects mysteries: two reactions of the different instruments standing in the different places are almost identical but they are shifted in time. The cross-correlation analysis indicates the high degree of the both curves similarity. The correlation coefficient in this case is $0.915 \pm 0.193$.

\section{DISCUSSION}

New phenomena that were unknown before the cooperative torsind/pendulum observations were found.

We do neither attempt to discuss these phenomena in detail nor to give them an explanation. The detailed theory for such analysis is not yet developed and the well-founded hypotheses are absent. Moreover, the detected effects themselves can contradict the logic of cause-and-effect relationships. This features cause skepticism of many researchers especially those who consider themselves to be classical determinists. The described results lead some scientists to believe that the equipment used is too simple to obtain results affecting the basics of physical science. Yes, the equipment is really simple. But this property provides its reliability.

The simultaneous measurements by several instruments yielded similar results with the high degree of similarity in many cases. It is known that if long series with hundreds and thousands of individual samples show the correlation coefficient close to one, then the random error of each series is negligible. When two or more instruments show the convergent result, then the results can be trusted. As can be seen from the given graphs, such cases were often observed in our practice. Our conclusion about the obtained results reliability becomes more stringent if we take into account the fact that not only the simultaneous readings of the different torsinds correlate but it also does the results of torsind and paraconical measurements as can be seen from the results of several solar eclipses. Particularly indicative in this respect is the observation of the eclipse on 26.01.2009. The coefficient of cross-correlation "pendulum-torsind" was as high as 0.921. It is not possible to explain such good coincidence of the curves shown in Fig. 7 by chance. We may only declare that the real events are reflected in these measurements. 
The experiments described above do not give an answer to the question "why?" The revealed temporal changes can hardly be explained in terms of electromagnetism and gravitation. We assume that the real effective cause lies outside these two fundamental interactions. That is why there is no adequate explanation for these changes within the framework of electromagnetic theory yet. We are mentioning electromagnetism only, as gravitation in any way can not be inured as a causal beginning, because both the torsion balance and the torsind structurally are not receptive to the change in gravipotential by definition.

In particular, it is necessary to answer the question of why the torsind's reaction can be both advanced and delayed. Or, why does the torsind rotate CW and CCW? Does this indicate that the Earth carries the torques of inverse polarities? Or the sign of polarity depends on other circumstances? Why in the last decade the intensity of the radiation that rotates the torsind disk increased by tens of times $[11,18]$ ? What penetrating power is this hypothetical solar radiation if it is felt by instruments being set in deep underground? All this is to be understood in future as we hope.

\section{CONCLUSION}

This article briefly describes the torsind, its features, and the results obtained with it. It seems that the rotation of the torsind disk has a much deeper reason than the obvious causes (changes in gravity, temperature, pressure).

The observed effects can't be explained within the framework of gravity or electromagnetism and it is assumed that they indicates to the manifestation of a new type of physical interaction and forces us to look for new fundamental connections in Nature.

The important observational argument has been obtained in favour of the fact that the unknown radiation acting on some mechanical devices (the torsind and pendulum) has neither electromagnetic nor gravitational nature. And if so, our planet is exposed to an unknown radiation. And the nature of this impact has not yet been studied and understood. At best, it can be studied and used by an expert in his competence. In the worst case, the influence of the new unknown raiation on the Earth can be decisive for existence of the planet and the entire earthly civilization.

\section{DATA AVAILABILITY}

All the experimental data that form the basis of this publication belong to the author and are published in open access journals (see References).

\section{CONFLICTS OF INTEREST}

N/A

\section{FUNDING STATEMENT}

This work was carried out within the framework of the current research plan of the Main Astronomical Observatory of the National Academy of Sciences of Ukraine.

There are no targeted grants, no sponsorship.

\section{ACKNOWLEDGMENTS}

The author thanks Professor E.A. Pokrovsky for fruitful help and advices. 


\section{REFERENCES}

1. M.F.C. Allais, "Movements of the Paraconical Pendulum and the Total Solar Eclipse of 30 June 1954", Proceedings of the French Academy of Sciences, 245, 1957, pp. 2001-2003.

2. E.Saxl, M.Allen. 1970 Solar Eclipse as 'Seen' by a Torsion Pendulum. Physical Review D, Vol. 3, No. 4, 1971, pp. $823-825$

3. T. Kuusela. New measurements with a torsion pendulum during the solar eclipse//General Relativity and Gravitation 24(5), pp.543-550, DOI: 10.1007/BF00760136

4. L.A. Savrov. Experimental research with short paraconical pendula of gravitational effects during solar eclipses. In: "Should the laws of gravitation be reconsidered?", Ed. Héctor A. Múnera, (Montreal: Apeiron 2011), pp. 149 - 164

5. S. W. Zhou, B J. Huang and Z. M. Ren The abnormal influence of the partial solar eclipse on December 24th, 1992, on the time comparisons between atomic clocks. Il Nuovo Cimento C (1995) 18: 223. https://doi.org/10.1007/BF02512022

5. Xin-She Yang, Qian-Shen Wang. Gravity Anomaly During the Mohe Total Solar Eclipse and New Constraint on Gravitational Shielding Parameter //Astrophysics and Space Science (2002), 282 (1), pp.245-253

6. Qian-shen Wang, Xin-she Yang, Chuan-zhen Wu, Hong-gang Guo, Hong-chen Liu, Chang-chai Hua. Precise Measurement of Gravity Variations During a Total Solar Eclipse // Physical review D: Particles and fields, 2010, 62 (4)DOI: 10.1103/PhysRevD.62.041101

7. A. Iovane. Simultaneous occurrence of periodic eclipse anomalies at distant sites // In "Should the laws of gravitation be reconsidered?", Ed. Héctor A. Múnera, (Montreal: Apeiron 2011), pp.175-190

8. Zhou, S. W. Huang, B. J.; Ren, Z. M. The abnormal influence of the partial solar eclipse on December 24th, 1992, on the time comparisons between atomic clocks. Nuovo Cimento C, 1995, Vol. 18C, N. 2, p. $223-236$.

9. Udem, T., Reichert, J., Holzwarth, R., Haensch, T., Kraemer, R., Hahn, J., Hammesfahr, J. Chronometry: Effect of the 1999 solar eclipse on atomic clocks. Nature 402, 749-750 (16 December 1999) | doi:10.1038/45442

10. Pugach A.F. The Torsind-A Device Based on a New Principle for Non-Conventional Astronomical Observations // International Journal of Astronomy and Astrophysics. - 2013, V.3, No. 2A, p. 33-38 doi:10.4236/ijaa. http: //www.scirp.org/ journal/ ijaa

11. Pugach A.F. Torsind as a Recorder of a Possibly New Energy (http: // www.vkingpub.com/ journal/ tepe) Thermal Energy and Power Engineering, 2013, V.2,No.4,p.129-133, http://www.vkingpub.com/ journal/ tepe

12. Goodey T.J., Pugach A.F., Olenici D. Correlated anomalous effects observed during the August 1st 2008 solar eclipse. http:// stoner. phys. uaic.ro/ jarp/ index.php/ jarp/ article/ view/ 40/22. Journal of Advanced Researches in Physics, 2010, V.1, No.2, ID 021007

13. A. F. Pugach and D. Olenici. Observations of Correlated Behavior of Two Light Torsion Balances and a Paraconical Pendulum in Separate Locations during the Solar Eclipse of January 26th, 2009// Advances in Astronomy, Volume 2012, Article ID 263818, 6 pages. http://dx.doi.org/10.1155/2012/263818

14. http://chronos.msu.ru/old/cabinets/nature ref/pugach/pugach nablydenia.pdf 
15. Dimitrie Olenici, Alexander F. Pugach, Ilie Cosovanu, Cezar Lesanu, Jean-Bernard Deloly, Danil Vorobyov, Alexander Delets, Stefan-Bogdan Olenici-Craciunescu. Syzygy Effects Studies Performed Simultaneously with Foucault Pendulums and Torsinds during the Solar Eclipses of 13 November 2012 and 10 May 2013. International Journal of Astronomy and Astrophysics, 2014, v.

16. Nikolsky, G. A., Pugach, A. F. (2016). Gravitational Lensing of Spiral Vortex Solar Radiation by Venus. Open Access Library Journal, 3, e2728. doi: http://dx.doi.org/10.4236/oalib.1102728. PP. 1-

17. Nikolsky G.A. The most likely sources of the Spiral Vortex Sun Radiation. Proceedings of the First Interregional Symposium "Ecology and Space" named by.Ya. Kondratiev, 8-9 February, 2012. Publ. St.Petersbourg Univ., 2012, pp. 374-387. (in Russian).

18. Pugach A.F. Diurnal Variations and Spikes by the Torsind Registered and Their Impact on the Accuracy of G Measurement (http: //www.scirp.org/ journal/ ijaa). International Journal of Astronomy and Astrophysics, 2015, 5, p.28-37 